\title{
Les non-usagers de l'internet. Axes de recherche passés et futurs
}

Non-users of internet. Past and future research directions

\section{Sally Wyatt}

Traducteur : Florence Waïtzenegger

\section{OpenEdition \\ Journals}

Édition électronique

URL : http://journals.openedition.org/questionsdecommunication/397

DOI : 10.4000/questionsdecommunication.397

ISSN : 2259-8901

\section{Éditeur}

Presses universitaires de Lorraine

\section{Édition imprimée}

Date de publication : 1 décembre 2010

Pagination : 21-36

ISBN : 978-2-8143-0056-9

ISSN : $1633-5961$

\section{Référence électronique}

Sally Wyatt, «Les non-usagers de l'internet. Axes de recherche passés et futurs », Questions de communication [En ligne], 18 | 2010, mis en ligne le 01 décembre 2012, consulté le 30 avril 2019. URL : http://journals.openedition.org/questionsdecommunication/397 ; DOI : 10.4000/

questionsdecommunication.397 


\section{LES NON-USAGERS DE L'INTERNET. AXES DE RECHERCHE PASSÉS ET FUTURS}

Résumé. - Cet article rassemble une partie des premiers travaux sur le non-usage de l'internet. La première partie est essentiellement conceptuelle et avait été présentée en 1999, au moment du pic de la première explosion des sites en « .com » et lorsque les attentes à l'égard de l'internet et les craintes en matière de fracture numérique étaient à leur apogée. Les auteurs démontrent comment le non-usage pouvait être un choix volontaire et n'aboutissait pas toujours à l'exclusion sociale, ni n'en était le produit. La seconde s'appuie sur une étude empirique qui s'est intéressée à la manière dont des personnes d'âge mûr trouvent des informations en matière de santé et les raisons pour lesquelles, dans ce cadre, ils utilisent ou non l'internet. La conclusion engage une réflexion sur des travaux récents traitant du non-usage de l'internet et propose des axes théoriques et empiriques pour les recherches futures.

Mots clés. - Internet, non-usagers, résistance, vie quotidienne, informations médicales, études scientifiques et techniques. 
Notre réflexion sur le non-usage de l'internet est née il y a environ une dizaine d'années en collaboration avec Graham Thomas et Tiziana Terranova alors que celui-ci et les premiers sites web en «.com » étaient en pleine expansion (Wyatt, 1999)'. À la fin des années 90 et au début du XXle siècle, la fracture numérique avait été l'objet de vastes enjeux politiques. Les décideurs politiques s'intéressaient aux individus, aux groupes sociaux et aux pays qui commençaient à connaître l'exclusion sociale, politique et économique, produite par l'absence de connexion numérique. Mais dès la fin des années 90, des données sont apparues montrant que le non-usage de l'internet n'était pas toujours perçu comme un problème par ceux qui n'y avaient pas accès et relevait parfois d'un choix (Katz, Aspden, 1998). Souhaitant remettre en question la doxa qui présuppose que chacun serait un usager en puissance, nous avions choisi comme point de départ de nous démarquer de l'idée dominante soutenant que le non-usage devrait toujours être perçu comme un manque et/ou un problème à résoudre. Nous cherchions également à comprendre la signification du non-usage de l'une des technologies majeures du XXe siècle dans les études scientifiques et techniques (Science and Technology Studies) et, plus généralement, dans les études concernant les nouveaux médias. La première partie de cet article résume ce travail pionnier sur le non-usage. Dans une seconde, nous reprendrons cette question de manière plus empirique. Nous examinerons le cas de personnes cherchant des informations sur la santé2. Mobilisent-elles à cet effet l'internet et comment s'y prennent-elles? Nous conclurons en présentant les termes d'un débat ouvert par des recherches récentes sur le non-usage.

\section{Le non-usage de l'internet}

À la fin des années 90, le non-usage de l'internet était perçu comme une question politique majeure et de nombreux débats à ce sujet ont été nourris par l'hypothèse selon laquelle le non-usage ou l'absence d'accès était un manque à combler ${ }^{3}$. Ainsi, en mars 2000, la réunion des chefs d'État ou de gouvernement de l'Union européenne de Lisbonne avait-elle été baptisée « Sommet dot. com », traduisant une prise de conscience de l'importance économique de la technologie de l'information et de la communication en général et de l'internet en particulier. L'objectif du Sommet était de réfléchir à l'accroissement de l'usage via la promotion de l'esprit d'entreprise, de la compétitivité et d'une

\footnotetext{
Cette recherche a obtenu l'appui financier du Conseil de la recherche économique et sociale du Royaume-Uni sous l'égide du Programme de recherche « La société virtuelle? » (1997-2002) dirigé par Steve Woolgar (numéro de subvention : LI 3225 I050).

${ }^{2}$ Ce projet a aussi obtenu l'appui financier du Conseil de la recherche économique et sociale du Royaume-Uni sous l'égide du Programme de technologies innovantes pour la santé (1997-2002) dirigé par Andrew Webster (numéro de subvention : L2 I 8252039).

3 Cette partie est fortement inspirée de Wyatt, Thomas et Terranova (2002) et Wyatt (2003). Elle s'appuie aussi sur la présentation faite en 1999 et signalée au début de cette contribution.
} 
économie dynamique fondée sur les connaissances. Chacun était présumé être un usager potentiel. L'accès à la technologie était nécessaire et souhaitable, son augmentation devenant le défi politique à relever afin de donner corps à son potentiel économique. Certains chefs d'État ont exprimé de vives inquiétudes, craignant le développement d'inégalités sociales si la « fracture numérique » devait augmenter. De telles préoccupations concernant l'exclusion sociale, louables au demeurant, sont toutefois fondées sur l'hypothèse que les « nantis » numériques seront dans une meilleure situation socio-économique que les « laissés pour compte ». Comme le souligne David Neice (2002:67)4, « ceux qui luttent pour l'élimination de la "fracture numérique" croient tout simplement que l'accès à [l']internet est toujours préférable à l'absence d'accès » Les raisons qui poussent les promoteurs privés de produits et des services en ligne à mettre en avant l'internet comme media universel sont évidentes, celles émanant de responsables politiques sont moins claires.

Le Sommet européen de mars 2000 est un exemple parmi d'autres du constat que feraient les politiciens et décideurs selon lequel le problème serait le manque d'accès à l'internet. Dans cette perspective, de nombreuses mesures ont été prises pour que soient réduits des coûts et la facilitation d'accès aux systèmes d'éducation et de formation. Il était supposé qu'en surmontant les freins à l'usage, les populations pourraient adopter la technologie sans réserve. L'accès à l'internet constitue nécessairement un bienfait, voire plus encore. Les hommes politiques espéraient que les populations utiliseraient leurs connaissances de l'internet et celles provenant du réseau pour créer de la richesse et de l'emploi et devenir des citoyens actifs, tout en sachant qu'ils pourraient également l'utiliser pour - par exemple - visionner des productions pornographiques ou pratiquer des jeux.

Au cours des quinze dernières années, la vie quotidienne a fait l'objet d'attentions particulières dans les études sur les technologies (Bakardjieva, Smith, 200 I ; Lie, Sørensen, 1996; Silverstone, Hirsch, 1992; Oudshoorn, Pinch, 2003). Plutôt que de focaliser les recherches sur les sites de création et sur les usages professionnels des technologies, les chercheurs se sont plus particulièrement intéressés aux pratiques quotidiennes au cours desquelles les usages et les significations sont discutés par des personnes « ordinaires ». Les usagers n'ont plus été perçus comme de simples récipiendaires passifs de la technologie, mais comme des acteurs importants et actifs dans le façonnement et la négociation du sens de la technologie. Ce qui est significatif tant pour la compréhension des processus de conception que pour la relation existant entre l'identité des technologies et leurs usagers. Inclure les usagers permet de surmonter les problèmes inhérents aux approches scientifiques, techniques et aux études d'innovation qui privilégient la place d'acteurs puissants dans la production des technologies comme les chercheurs, les ingénieurs, les hommes politiques et les financiers. Les usagers s'immiscent de plus en plus dans les études technologiques pour contrebalancer

\footnotetext{
${ }^{4}$ «It is simply presumed by those advocating the elimination of the "digital divide" that having Internet access is always better than lacking it $\gg$.
} 
la place prise par les producteurs, laquelle est présente dans une grande partie de la littérature scientifique, même si toute catégorie provoque des exclusions. Rétablie par la reconnaissance de l'importance de l'usage et des usagers, la dialectique existant entre production et consommation peut toutefois faire surgir un autre problème. En effet, focaliser l'attention sur l'usage signifie l'acceptation implicite des promesses de la technologie et des relations capitalistes de sa production. Par conséquent, les usagers devraient être appréhendés en relation avec un autre groupe, moins visible, celui des non-usagers.

À la fin des années 90 , les rares études portant sur le non-usage ont identifié comme raisons majeures de celui-ci le coût (du matériel informatique de même que celui de la maintenance et de l'accès) et le manque de besoin ou d'intérêt (CyberDialogue, 2000). Sur la base de deux enquêtes téléphoniques nationales aléatoires menées aux États-Unis, James Katz et Philipp Aspden (1998) suggèrent l'existence de figures du non-usage. Les personnes ayant cessé l'usage de l'internet étaient plutôt pauvres et peu diplômées. Celles ayant été initiées par l'intermédiaire d'un membre de la famille ou d'amis sont plus susceptibles « d'abandonner » que celles qui ont appris par elles-mêmes ou qui ont reçu une formation spécifique au travail ou à l'école. Les adolescents sont davantage susceptibles d'abandonner que les individus âgés de plus de vingt ans. Les motifs « d'abandon » varient en fonction de l'âge. Les personnes plus âgées se plaindraient volontiers du coût et des difficultés d'utilisation rencontrées, tandis que les plus jeunes arrêteraient suite à une perte d'accès ou par manque d'intérêt. James Katz et Philipp Aspden ont attiré l'attention sur cette catégorie importante de non-usagers. Bien que restant dans une approche fonctionnaliste, leurs justifications du non-usage mettaient l'accent sur des questions de coût et d'accès. Le recours au terme « abandonniste » est plutôt péjoratif, et suggère que l'usage serait préférable au non-usage. Comme l'affirme Ronald Kline (2003), aborder la résistance à la technologie dans une perspective fonctionnaliste conforte les interprétations des promoteurs de son succès. Dans son étude sur la résistance au téléphone et à l'électrification aux États-Unis au début du $X X$ e siècle, il définit la résistance du point de vue des acteurs contemporains et démontre comment celle-ci peut elle-même induire un changement social et technique.

Plusieurs catégories de non-usage sont à différencier. Comme le souligne Martin Bauer (1995: | 4- 15), une différence existe entre « comportement d'évitement » passif et résistance active. De même, il faudrait distinguer le non-usage de l'ensemble du système technologique (comme l'internet) du non-usage d'aspects particuliers (Miles, Thomas, 1995 : 256-257). Dans une précédente taxinomie, nous avons identifié quatre types de non-usagers (Wyatt, Thomas, Terranova, 2002 : 36). Nous sommes parvenu à deux dimensions dont la première distinguait le non-usage volontaire de celui involontaire, et la seconde qui opposait les individus n'ayant jamais eu un accès à l'internet à ceux qui en avaient déjà eu un et l'avaient perdu pour une raison ou une autre. Cela a permis d'identifier quatre catégories : les résistants, les « abandonnistes » (rejectors), les exclus et 
les expulsés. Les résistants n'ont jamais eu accès et ne l'ont jamais souhaité. Les « abandonnistes » ont essayé mais ont volontairement renoncé. Les exclus n'ont jamais eu d'accès internet, là encore sans que cela relève de leur propre choix. Les expulsés ont eu un accès à l'internet mais l'ont perdu, après avoir terminé leurs études ou changé de travail; une perte ne relevant certainement pas d'un choix volontaire. Les exclus et les expulsés sont les catégories à destination desquelles les politiques et les pourvoyeurs de technologie orientent leurs arguments. Nous estimions que les résistants et les abandonnistes étaient ignorés quand bien même étaient-ils importants à plus d'un titre. Même si l'on accepte l'idée selon laquelle l'inclusion numérique déboucherait d'une façon ou d'une autre sur l'inclusion sociale, l'analyse des raisons pour lesquelles certains individus n'utilisent pas les technologies numériques pourrait éclairer notablement les responsables politiques et les fournisseurs. Peut-être que les non-usagers trouvent les applications actuelles ni utiles ni agréables, alors que des applications futures - non encore découvertes - pourraient les entraîner dans la sphère numérique? À ce titre, les non-usagers peuvent-ils également modeler les technologies du futur? Toutefois, les résistants et les « abandonnistes 》 sont plus importants par le défi qu'ils lancent à l'impératif technologique (Ellul, 1964, 1980; Uitonen, 2003), voire à l'impératif numérique et à l'idée qu'il existerait une seule logique numérique pour tous les individus, les organisations et les pays. En d'autres termes, les personnes qui choisissent de ne pas utiliser des technologies numériques rappellent que les choses « peuvent avoir été autrement 》(Bijker, Law, 1992 : 3). Elles témoignent aussi du fait que, dans certains cas, l'exclusion numérique ne signifie pas toujours exclusion sociale.

Dès que l'on inclut « l'ancien usager » comme « l'usager actuel » et « celui qui n'a jamais été usager », la notion « d'usager » s'en trouve déconstruite. Que signifie exactement être usager? Est-il possible de distinguer non-usagers et non-possesseurs? La fréquence d'usage, les moyens d'accès et l'éventail des services utilisés sont des considérations importantes pour la conceptualisation de l'usage de l'internet. L'« usager » de l'internet doit être conceptualisé selon un continuum qui présente des variations dans l'intensité et les formes de participation. Les différentes modalités d'usage devraient être appréhendées selon les types d'usagers, mais aussi en relation avec la diversité des trajectoires temporelles et sociales. Ces dernières comprennent les changements de mode de vie déterminés par des processus tels le vieillissement, le changement de travail, les études et la mobilité géographique. L'usage de l'internet englobe non seulement divers types d'usage, mais aussi la possibilité d'inverser et de changer la direction des figures d'usage individuelles et collectives. Outre les variables démographiques habituelles, des précisions sur la fréquence et la nature de l'usage aideraient à brosser un tableau plus complet des multiples usages et usagers. De nombreux auteurs ont souligné les façons dont les producteurs et les concepteurs de technologie dépeignent l'usager singulier comme indifférencié ou la pluralité d'usagers comme groupe homogène dans la « e-methodology », adoptant eux-mêmes le paradigme de l'usager (Lindsay, 2003). Incorporer de la 
sorte une variété de non-usagers permet également d'obtenir une description et une analyse plus subtiles de la multiplicité des usagers.

La reconnaissance de l'existence des non-usagers amplifie certains problèmes méthodologiques dans l'analyse du changement social et technique. Nous avons déjà mentionné l'importance d'inclure les usagers dans les études technologiques afin d'éviter les pièges consistant à suivre seulement les acteurs puissants. Une autre façon de les éviter est de considérer les non-usagers et les anciens usagers comme des groupes sociaux pertinents, comme des acteurs qui pourraient exercer une forte influence. Mais il faudra faire face à d'évidentes difficultés de méthode liées au repérage des non-usagers. Ceux-ci pourraient constituer un groupe peu cohérent, chacun ayant peut-être des raisons fort différentes de ne pas utiliser l'internet. Ce groupe invisible exprime à nouveau les difficultés posées par une surinterprétation de la maxime « suivez les acteurs » (Latour, 2005)5. Pour la partie suivante, nous avons suivi des personnes qui, s'informant sur leurs problèmes de santé et les traitements possibles, ont essayé d'utiliser l'internet.

\section{Une étude empirique du non-usage}

Une grande partie de la littérature scientifique de la fin des années 90 et du début des années 2000 portant sur la fracture numérique - nos travaux compris - est restée à un niveau abstrait, se focalisant sur l'usager générique ou idéal sans examiner les pratiques quotidiennes de recherche d'information des individus

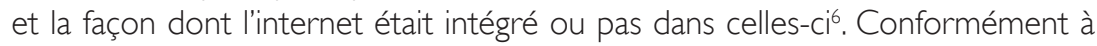
l'approche des usages et des usagers mentionnée ci-dessus, nous nous sommes aussi engagé dans l'étude des usages et des usagers dédiés à la recherche d'informations médicales. Notre approche est pleinement placée sous les auspices des travaux de Pierre Bourdieu (1977, 1984) et de Michel de Certeau (1984) en prenant en charge à la fois la nature symbolique et matérielle des artefacts et leurs rôles dans la production de relations sociales. Nous partageons leurs préoccupations sur l'inégalité et sa reproduction de même que sur la perception de la nature active de la consommation et les possibilités de résistance que les individus peuvent exercer dans leur vie. Dans les pages qui suivent, nous nous intéresserons aux différences générationnelles et de genre, telles qu'elles s'inscrivent dans les pratiques quotidiennes de l'usage de l'internet.

Dans la lignée de Sherry Turkle (1995) et de Maria Bakardjieva (2003), nos recherches ont démarré par l'usage individuel de l'internet. Alors que ces chercheurs se sont concentrés respectivement sur la manière dont les individus utilisent l'internet pour les jeux et les processus de familiarisation, nous avons

\footnotetext{
${ }^{5}$ Alors que l'analyse est guidée par la théorie de l'acteur-réseau, dans la partie suivante « acteur » sera pris au sens sociologique le plus courant, à savoir celui de l'acteur individuel humain.

${ }^{6}$ Cette partie s'inspire de Wyatt, Henwood, Hart, Smith (2005).
} 
cherché à savoir si et comment, dans les pratiques quotidiennes, l'internet était mobilisé pour recueillir des informations médicales. Reconnaissant que les individus disposent d'un large éventail de sources d'informations, comme les médias de masse, les professionnels de santé, la famille et les amis, notre étude interroge la manière avec laquelle l'internet s'inscrit dans ces paysages informatifs et comment il s'intègre dans les pratiques changeantes des individus.

Cette recherche s'appuie sur le cas d'hommes et de femmes résidant dans le sud-est de l'Angleterre et qui cherchaient à s'informer sur des questions de santé liées au vieillissement, plus particulièrement sur le défaut d'érection masculine et la ménopause ainsi que leurs traitements les plus courants que sont le Viagra et la THS (thérapie hormonale de substitution). Ménopause et dysfonctionnement érectile ne sont ni des problèmes aigus ni des problèmes mettant en jeu le pronostic vital, les symptômes pouvant durer longtemps. Ainsi les personnes touchées disposent-elles de temps pour s'informer sur leur état et les possibilités de traitements. Nous examinons ici comment l'internet peut être utilisé comme un média donnant accès et communiquant des informations médicales.

Des entretiens portant sur les raisons ayant conduit les sujets à penser à la THS ou au Viagra ont été menés auprès de 32 femmes et 15 hommes. II leur a été demandé où et comment ils recherchaient et se procuraient ces informations, comment ils les interprétaient et leur donnaient du sens (par eux-mêmes ou grâce à des échanges avec d'autres personnes, y compris lors de consultations avec des professionnels). Aux personnes ayant recours à l'internet, il a été demandé depuis quand et dans quels buts ils l'utilisaient. En cas d'utilisation de l'internet pour la recherche en ligne d'informations médicales, ont été demandées des précisions sur la façon dont ils s'y étaient pris ainsi que leur opinion sur les avantages et inconvénients de ce support comme source d'information. Près de la moitié des participants - soit 16 femmes et 5 hommes - ont fait l'objet d'une seconde enquête, six à neuf mois après le premier entretien afin de voir si leur état avait évolué et s'il y avait eu des changements dans leurs méthodes de recherche et de traitement de l'information. Les femmes ont été sélectionnées par l'intermédiaire d'un médecin de famille ou de la clinique gynécologique d'une ville du sud-est de l'Angleterre. Les hommes ont été choisis via une clinique urologique, un service de conseils psychosexuels pour hommes souffrant de dysfonctionnement érectile ou une clinique pour diabétiques. Seuls les femmes et les hommes supposés avoir pris ou avoir récemment cessé de prendre de la THS ou du Viagra ont été sollicités. Les entretiens d'une durée allant d'une à deux heures se sont tenus entre novembre 200 I et janvier 2003. Les entretiens ont été enregistrés et totalement retranscrits. La plupart ont eu lieu chez les participants, à quelques exceptions près, certains s'étant déroulés dans des bureaux au sein des structures de soins. L'âge des femmes interrogées était compris entre 39 et 73 ans avec un âge moyen de 55 ans. Parmi ces 32 femmes, 18 vivaient en couple. Les hommes étaient plus âgés : 54 à 8 I ans pour un âge moyen de 66 ans. Parmi eux, dix vivaient en couple. L'échantillon provenait de différents groupes socio- 
économiques avec un niveau de formation et de qualification variable. La plupart des sujets, mais pas tous, étaient hétérosexuels et majoritairement britanniques blancs.

Les sources d'information potentielles sont gigantesques. Comment les participants s'informent-ils sur des questions médicales? Globalement, tous ont puisé, de manière plus ou moins active, dans diverses sources. Pour les hommes et les femmes, le médecin de famille est la source la plus importante. Puis viennent les membres de la famille, en général des femmes, deuxième source la plus souvent citée à laquelle on peut ajouter des amis, des pharmaciens et des professionnels de médecine douce. Les médias consultés sont les revues, la télévision, le Web, les livres traitant du développement personnel, les journaux et autres publications comme des brochures provenant de pharmaciens ou fournies avec les médicaments par les laboratoires pharmaceutiques. Ce qui différencie le plus les hommes des femmes est avant tout le large réseau social sur lequel s'appuient celles-ci pour parler de leurs problèmes de santé et qui englobe la famille, les amis, les voisins et les collègues alors que les hommes s'adressent principalement à leurs médecins et à leurs partenaires sexuels.

Cette vue d'ensemble offre des pistes de compréhension des expériences vécues au quotidien par des personnes essayant d'accepter les problèmes dus au vieillissement et cherchant à s'informer à ce sujet. Approfondissons cette question afin de comprendre comment l'internet s'inscrit dans un paysage d'informations et voir dans quelle mesure la fracture numérique serait une expérience vécue en nous intéressant plus particulièrement aux conditions d'accès. Sur les 15 hommes enquêtés, 9 disposaient d'une connexion, dont un sur son seul lieu de travail sans pour autant être autorisé à l'utiliser à des fins personnelles. Quelques autres étaient en situation transitoire. Un homme avait déménagé récemment et n'avait pas encore déballé son ordinateur ni entrepris les démarches auprès de son fournisseur d'accès. Un autre possédait un téléphone portable nouveau permettant un accès internet qu'il ne savait toutefois pas encore faire fonctionner. Finalement, un autre avait eu accès au Web par l'intermédiaire du travail de son épouse où son patron l'avait aidé à se renseigner sur son problème mais cela est resté une expérience unique qui ne l'a pas incité à rechercher un accès régulier à l'internet. Sur les 32 femmes, 24 possédaient une connexion internet au domicile et/ou au travail. Parmi les enquêtés, huit hommes et 20 femmes utilisaient l'internet à la maison ou au travail mais tous ne s'en servaient pas pour chercher de l'information médicale. Seuls trois hommes et I 8 femmes l'ont fait.

Toutes les femmes disposant d'une connexion à domicile n'en font pas pour autant l'usage. C'est le cas de Janet dont l'ordinateur familial est essentiellement utilisé par le fils. Barbara explique également comment son fils monopolisait l'ordinateur jusqu'à ce qu'il parte à l'université. Mais son fils n'a pas nettoyé l'ordinateur avant son départ. Ce dont elle parle avec émotion : 
Les non-usagers de l'internet. Axes de recherche passés et futurs

« J'ai eu pendant longtemps à la maison un fils adolescent qui monopolisait internet et ne voulait pas me montrer comment cela marchait parce qu'il disait que j'étais trop lente. II vient de partir à l'université maintenant, alors j'ai vraiment essayé cette semaine de le mettre en route et de l'arranger mais c'est un tel bazar... Ce qui arrive, c'est que quand je l'allume, il met 20 minutes à démarrer... parce qu'il y a tellement d'affaires dedans, avec des économiseurs d'écran et ce truc et celui-là... J'ai plusieurs voisins qui me promettent toujours de venir m'aider, donc je vais y arriver. J'ai suivi un cours de base. Mais je n'aime pas. Je déteste cela... Cela ne m'intéresse pas. Je pense simplement que c'est très ennuyeux. L'idée de le mettre en route me fait autant envie que de repasser $\gg$.

Bien que reléguée par son fils au rang de vieille dame qui ne s'intéresse pas à l'usage des ordinateurs et qui ne possède pas les compétences nécessaires, Barbara résiste à cette image. Elle comprend pourquoi la machine est ralentie. En demandant conseil à des voisins et en suivant des cours, elle a entrepris de s'informer davantage et d'enrichir ses compétences. Néanmoins, elle garde une forme d'ambivalence et se réfère à son rôle domestique lorsqu'elle exprime un manque d'intérêt et un sentiment d'ennui. D'autres femmes parlent plutôt de leurs partenaires masculins que de leurs fils et racontent que leurs conjoints possèdent des ordinateurs avec un accès internet à domicile qu'elles n'utilisent jamais. Ainsi Betty explique-t-elle qu'il y avait avant un ordinateur connecté chez elle mais qu'il appartenait à son ex-conjoint :

« II l'a emporté lorsqu'il est parti. Je n'en ai plus... II l'utilisait surtout pour le travail... J'avais pensé m'en servir mais malheureusement nous nous sommes séparés ». Frieda, elle, parle de son mari : « II possède quatre ordinateurs situés à l'étage. II les utilise pour son travail. I| est à la retraite... || est architecte... || fait des rajouts aux maisons et modifie les intérieurs et des tas de choses comme cela, mais il peut tout faire sur l'ordinateur. Moi, je ne saurai même pas l'allumer ».

Ces exemples accréditent l'idée selon laquelle il convient d'interpréter soigneusement les résultats d'enquête à large spectre en matière d'accès à l'internet. Ces femmes vivent et ont vécu avec des ordinateurs connectés depuis leur domicile, mais n'en ont pas l'usage pour différentes raisons. Accéder à l'internet ne se réduit pas à une simple question d'équipement et de connexion, ou de formation et d'obtention de compétences nécessaires à l'utilisation de l'équipement. L'accès présuppose l'existence d'un sentiment de confort par rapport à la technologie et suppose de ne pas en avoir peur. Cela suppose également que la répartition des tâches ménagères permette aux femmes de se sentir concernées par les machines. En effet, dans de nombreux ménages, perdure un modèle traditionnel de répartition sexuée des tâches. Les partenaires masculins et les fils monopolisent l'ordinateur, obligeant les femmes à renoncer à l'utiliser ou les contraignant à trouver un autre lieu pour y avoir accès. Ce sexisme ne correspond cependant pas toujours aux stéréotypes comme dans le cas de Peter (66 ans), séparé de sa femme. II a quatre fils dont deux qui vivent avec lui. Peter a quitté l'école avant d'avoir 16 ans et s'est occupé d'un petit commerce de détail jusqu'à sa retraite qu'il a prise peu de temps avant l'entretien. Son foyer possède un ordinateur connecté à internet utilisé par ses fils. Ressentant une certaine pression à devenir usager il déclare : « Je ne l'utilise pas. Je n'en ai pas 
envie... Je devrais davantage utiliser l'ordinateur, tout simplement je, je ne sais pas, je n'en ai simplement pas envie. Je suis paresseux pour cela. Ce n'est pas mon truc. Je préfère prendre le téléphone et appeler quelqu'un plutôt que lui envoyer un courriel qui me prend trop de temps ». Lorsqu'on l'interroge sur de précédentes expériences d'utilisation du courriel, il répond: « Je ne me débrouille pas bien du tout. Je rate plutôt tout. Soit je perds ma lettre d'une façon ou d'une autre, j'appuie sur le mauvais bouton ou cela ne passe pas correctement. J'ai toujours des tonnes d'ennui avec cela ». La préférence de Peter pour l'immédiateté et la présence apportées par le téléphone ainsi que son incompétence avouée dans le traitement des courriels conduisent à penser que les facteurs liés à l'âge et à la génération - auxquels il faut ajouter le genre - jouent un rôle important dans les expériences personnelles à l'égard de l'usage de l'internet. L'histoire de Victor défie encore davantage le stéréotype de la masculinité à propos de la technologie. Victor a 59 ans. II est marié et a trois enfants adultes. II a un emploi de direction et une formation de troisième cycle. Depuis un an environ, il souffre d'un manque de libido. Certes, il a des érections mais ne ressent aucune envie d'avoir une activité sexuelle. Cela le dérange beaucoup bien que son épouse n'en fasse pas état. La prise de Viagra lui a permis d'avoir des érections mais ne lui a pas donné envie d'avoir des relations sexuelles. Victor a d'abord parlé de ses problèmes à son épouse, puis à des professionnels de la santé. II ne consulte pas de guides pratiques de santé mais feuillette les pages consacrées à la santé dans les tabloïdes et les journaux sérieux. II s'intéresse aux panneaux affichés dans les salles d'attente et a tendance à prendre les informations au pied de la lettre. II n'a jamais eu à gérer des informations contradictoires en matière de santé. II aime discuter avec son épouse car cela lui permet d'explorer toutes les possibilités et/ ou de confirmer ce qu'il ressent. II n'entreprend aucune recherche particulière avant d'aller consulter son médecin. Victor n'a jamais utilisé l'internet mais envisage de se procurer un ordinateur domestique à des fins professionnelles. Cette partie de l'entretien est restituée ci-dessous :

« Enquêteur (E) : Avez-vous le moindre accès à internet en ce moment?

Victor $(V)$ : Je pourrais avoir accès grâce à ma fille ou à son petit copain.

$\mathrm{E}:$ Mais vous ne l'utilisez pas?

$\checkmark:$ Non

$E$ : Est-ce que vous vous en êtes déjà servi?

$\checkmark:$ Non

$E$ : Mais, vous y pensez, n'est-ce pas?

$\checkmark:$ À contrecœur!

$E$ : Pourquoi dites-vous « à contrecœur" ...?

$\checkmark$ : Je trouve qu'il est difficile de se familiariser avec les tâches techniques.

$\mathrm{E}:$ Ce n'est pas votre genre?

$\checkmark:$ Non

E : Alors pourquoi pensez-vous l'utiliser maintenant?

$\checkmark$ : Je pense que si je pouvais le maîtriser cela faciliterait beaucoup mon travail à temps partiel.

E: Pensez-vous que vous pourrez envisager de chercher de d'information sur la santé sur internet?

$\checkmark$ : Je pense que c'est peu probable.

E : Pourquoi? 
$\checkmark$ : Parce que je pense que je préfèrerais chercher dans un livre si je le veux vraiment ou en parler au médecin.

E : Je me demande pourquoi cela pourrait être le cas.

$\checkmark$ : [énervé] Les ordinateurs ne m'intéressent pas. Je veux juste poursuivre ma vie le plus tranquillement possible. Je ne vois pas l'avantage que cela me donnerait. Je préfèrerais l'apprendre de source sûre et en parler avec quelqu'un plutôt que me perdre dans le cyberespace. Cela me parait sacrément évident, et je pense que faire cette recherche est une perte de temps ».

Poussé par l'enquêteur à être plus explicite,Victor est clairement sur la défensive lorsqu'on évoque son non-usage de l'internet. Toutefois, son hostilité aux ordinateurs, à l'internet et à notre recherche semble sincère. Deux interprétations sont possibles. II peut s'agir d'un défi à la relation habituelle entre sexe masculin et technologie (Faulkner, 2000; Lie, 2003; Wajcman, 2004) qui présuppose que les hommes auraient un accès privilégié à la technologie et aux compétences techniques. Manifestement, Victor ne pense pas être privilégié, ni à l'égard des ordinateurs ni par rapport aux autres tâches techniques plus générales qui lui semblent toujours difficile à appréhender. Ensuite, il nous semble que la frustration à l'égard des ordinateurs, de l'internet et de l'enquêteur est plus facile à exprimer que l'impossibilité d'avoir des rapports sexuels. La masculinité et la compétence technique vont de pair. L'aptitude à utiliser la technologie et à avoir des rapports sexuels étant souvent associées à la masculinité, on peut penser que sa frustration en matière sexuelle se retrouve dans son rapport à la technique. Peter est un « abandonniste », membre de la deuxième catégorie de non-usagers décrite précédemment. Sur la base d'une faible expérience, Peter estime que l'internet, tout du moins le courriel, serait plutôt ennuyeux. II l'a essayé mais pense que cela ne lui correspond pas car il préfère l'immédiateté offerte par d'autres moyens de communication. Quant à Victor, il constitue un bel exemple de « résistant », correspondant à la première catégorie de non-usagers définie plus haut. II n'éprouve aucun intérêt personnel à utiliser l'internet. En ce qui le concerne, il existe d'autres façons tout à fait satisfaisantes de s'informer.

Cette étude portant sur des hommes et femmes d'âge mûr qui cherchent de l'information sur la ménopause et les problèmes d'érection a permis de cibler un groupe d'usagers relativement sous-étudié. Le recours à une approche s'appuyant sur des expériences de vie quotidienne des individus par rapport à l'internet et sur leur recherche d'information médicale permet de dépasser de façon fructueuse l'immobilisme et la polarisation auxquels renvoie le concept de fracture numérique. La fracture numérique ne saurait uniquement être appréhendée en termes de « nantis » et de « laissés pour compte ». Bien que Victor n'utilise ni ne dispose d'un accès à l'internet, les autres sujets devraient paraitre comme étant des « nantis » dans les statistiques comptabilisant les personnes possédant un accès à l'internet domestique. Nous avons montré que « l'accès » ne relève pas simplement d'une question de branchement. Les données disponibles en la matière gênent la mise en évidence de la complexité des relations domestiques. Bien qu'utilisant l'internet au travail à des fins personnelles, Janet le fait peu chez elle en raison de la mainmise de son fils. D'autres personnes interrogées, hommes comme femmes, ont mentionné qu'ils 
avaient chez eux des PC branchés mais qu'ils ne se connectaient pas, en général parce que les ordinateurs étaient monopolisés dans l'espace domestique par les fils ou les partenaires masculins. Accéder à l'internet nécessite bien plus qu'une proximité avec un bon équipement: il relève aussi des rapports de genre et intergénérationnels qui façonnent le contexte dans lequel les individus interagissent ou n'interagissent pas avec l'internet dans leur vie quotidienne. Les catégories auparavant élaborées sont utiles pour comprendre la résistance à la fois passive et active (Bauer, 1995; Rogers, 1986) aussi bien que les abandons (Katz, Aspden, 1998). Les histoires de Peter et de Victor invitent à repenser les prétentions optimistes et universalistes établies à l'égard de l'internet. Ces expériences conduisent également à revoir la relation structure-agent (Giddens, 1984). Comme Peter et Victor ignorent relativement tout de l'internet et de ses possibilités, peut-on pour autant dire qu'ils pèchent par manque d'engagement? Même si leurs comportements correspondent davantage à une esquive passive, les responsables politiques et les fournisseurs d'accès le perçoivent comme résistance à la technologie.

\section{Conclusion}

Nos premières recherches sur le non-usage ont surtout constitué en une expérience intellectuelle menée contre les nombreuses revendications grandiloquentes proclamées lors du changement du siècle et une réponse critique à la trop grande simplification du débat politique sur la fracture numérique. La résurgence de visions emphatiques prononcées autour du Web 2.0 et des réseaux sociaux montre combien le scepticisme analytique (Woolgar, 2002) est indispensable. Même si les applications et les interfaces peuvent avoir changé, les nombreuses revendications et promesses du Web 2.0 semblent véritablement familières. L'usage de l'internet dans toutes ses formes par les individus, les organisations et les pays est considéré comme la norme, le non-usage étant perçu comme une insuffisance à corriger ou comme besoin à combler. L'hypothèse sous-jacente est que l'accès à la technologie est évidemment souhaitable, la question étant de savoir comment l'augmenter. Les solutions préconisent parfois la mise en place d'investissements en matière d'infrastructure et dans l'éducation publique afin de surmonter l'ignorance et la peur, ou dans la formation et la standardisation pour améliorer l'usage. L'abandon de la technologie par des personnes informées et volontaires n'est pas mentionné. Cette invisibilité reflète la persistance de la domination d'une lecture vertueuse du progrès technologique, de la part des responsables politiques mais aussi au sein de la communauté scientifique.

Quelle sera la prochaine analyse théorique et empirique du non-usage? Constatons, d'abord, que si la lutte contre la fracture numérique n'est plus prioritaire dans les agendas politiques, pour autant, le non-usage subsiste. Avec l'amélioration des taux de connexion dans les pays industrialisés, la volonté de 
réduire la fracture numérique s'est apparemment estompée. Pourtant, au niveau mondial, la fracture demeure. En 2007, les pays « développés » comptaient 62 internautes pour 100 habitants contre seulement 17 dans les pays « en voie de développement » (ITU, 2009). L'écart reste toujours substantiel bien qu'il se soit réduit au cours des premières années du XXle siècle. Ces statistiques cachent également des différences considérables au sein des pays développés et en voie de développement. C'est ainsi que l'on compte moins I \% d'internautes dans les pays africains comme le Burundi, le Congo et l'Éthiopie alors qu'il y en a $32 \%$ au Maroc. De fortes disparités existent au sein même de l'Union européenne : les Pays-Bas, la Suède et le Danemark possèdent plus de 80 internautes pour 100 habitants alors que le Portugal et l'Italie en auraient moins de 50. L'accès aux ressources numériques reste un problème majeur accentué au niveau mondial dans les pays les plus pauvres par d'autres difficultés liées aux infrastructures comme l'approvisionnement en électricité et l'éducation. II est également nécessaire de prendre en compte les clivages dans la production du contenu en ligne et dans les infrastructures. Malheureusement, tous les pays ne disposent pas des données les plus récentes. Parmi les pays les plus riches du monde, il reste encore d'énormes disparités en termes de production de contenu (sites web) et de matériel informatique (brevets). Le nombre moyen de sites web pour I 000 habitants était de 32 pour l'ensemble des pays de l'OCDE alors qu'il était seulement de 10,5 en France et de plus de 80 en Allemagne (nationmaster. com). Alors que la diffusion devrait vraisemblablement se poursuivre, elle n'est pas conforme au rythme exponentiel prédit lors de la décennie précédente et, suivant la même évolution que d'autres technologies, l'internet pourrait également atteindre un niveau plateau qui serait bien loin d'une diffusion et d'un accès généralisés (Thomas, Wyatt, 2000). En deuxième lieu, plusieurs chercheurs ont relevé le défi empirique d'étudier le non-usage sous différentes perspectives. Dans la recherche par enquêtes, le non-usage n'est plus simplement évoqué pour compléter les calculs, mais il est devenu une catégorie à part entière. C'est ainsi que Ulrich Riehm et Bettina Krings (2006) ont montré à travers une enquête conduite en Allemagne en 2004 que parmi les $45 \%$ de la population qui étaient des non-usagers, $35 \%$ étaient des résistants, $25 \%$ des « abandonnistes », $20 \%$ des exclus et autant d'expulsés. Une enquête menée aux États-Unis a mis en évidence le fait que plus de la moitié des non-usagers sont des résistants ou des « abandonnistes » (Lenhart et al, 2003). Agnetha Broos (2006) a mis en évidence un groupe significatif de jeunes Flamands qui, estimant que l'internet n'est plus « branché », s'occupent avec d'autres passe-temps d'adolescents. Cependant, comme le montre cette contribution, les enquêtes de grande envergure doivent être complétées par des analyses plus fines portant sur les raisons qui poussent les individus à utiliser ou non l'internet dans leur vie quotidienne et sur la façon dont les figures d'usages ont évolué au cours du temps. Pour développer du matériel de formation adapté, Mike Cushman et Ela Klecun (2006) ont choisi comme point de départ de dépasser le cadre des explications socio-économiques du non-usage dans une recherche-action identifiant les raisons qui poussent à ne pas utiliser l'internet. Neil Selwyn, Stephen Gorard et John Furlong (2005) 
combinent des méthodes qualitatives et quantitatives pour comprendre qui sont les usagers et les non-usagers de l'internet dans la vie quotidienne.

Finalement, un cadre conceptuel plus dynamique et plus élaboré est nécessaire. Plutôt que de rester dans une vision manichéenne opposant usagers et nonusagers, l'usage des technologies numériques doit être conceptualisé à travers un continuum et selon divers degrés et types d'implication qui pourraient évoluer en fonction de l'éducation, de l'emploi, des enfants et des déménagements. Le nonusage n'est pas la seule pratique à déchiffrer. De façon symétrique, l'usage se doit également d'être explicité et ne devrait pas être considéré comme une pratique normale et tenue pour acquise. Les catégories doivent également être affinées afin d'intégrer non seulement l'abandon, la résistance, mais aussi « l'usage sélectif 》 (selective use) (Oudshoorn, 2008), « l'usage de remplacement » (surrogate use) (Selwyn, Gorard, Furlon, 2005), « l'usage forcé » (forced use), « l'usage réticent » (reluctant use) et « l'usage partiel » (partial use). Ces catégories plus nuancées rappellent que l'usage et le non-usage ne relèvent pas simplement des choix individuels et volontaristes. En effet, les résultats qui ressortent de l'analyse des personnes cherchant des informations médicales de même que ceux provenant de recherches plus récentes montrent que les choix des individus sont enracinés dans l'économie morale du ménage, dans les relations sociales et culturelles ainsi que dans les relations de production.

Traduit de l'anglais par Florence Waïtzenegger (Marseille)

\section{Références}

Bakardjieva M., 2003, «Virtual togetherness, an everyday life perspective », Media, Culture \& Society, 25, 3, pp. 29I-313.

Bakardjieva M., Smith, R., 200 I, «The internet in everyday life : computer networking from the standpoint of the domestic user », New Media \& Society, vol. 3, I, pp. 67-83.

Bauer M., 1995, «Resistance to new technology and its effects on nuclear power, information technology and biotechnology », pp. I-4I, in : Bauer M., ed., Resistance to new technology, Cambridge, Cambridge University Press.

BijkerW., Law J., 1993, « General introduction », pp. I - I 4, in : Bijker W., Law J., eds, Shaping Technology/Building Society: Studies in Sociotechnical Change, Cambridge, MA, MIT Press.

Bourdieu P., 1977, Outline of a theory of practice, trad. du français par R. Nice, Cambridge, Cambridge University Press.

— 1984, Distinction : A social Critique of the Judgement of Taste, trad. du français par R. Nice, London, Routledge \& Kegan Paul.

Broos A., 2006, De digitale kloof in de computergeneratie : ICT-exclusie bij adolescenten, PhD dissertation, Katholieke Universiteit Leuven.

Certeau M. de, 1984, The practice of everyday life, trad. du français par S. Rendall, Berkeley, University of California Press. 
Les non-usagers de l'internet. Axes de recherche passés et futurs

Cushman M., Klecun E., 2006, « How (can) non-users engage with technology : Bringing in the digitally excluded », pp. 347-364, in : Trauth E., Howcroft D., Butler T., Fitzgeral B., Gross J., eds, Social inclusion: Societal and organisational implications for information systems, Boston, Springer.

Cyber Dialogue, 2000, « Cyber Dialogue study shows U.S. Internet audience growth slowing 》. Accès : http ://www.cyberdialogue.com. Consulté le 30/03/2000.

Ellul J., 1964, The technological society, trad. du français par J. Wilkinson, New York, Random House.

— 1980, The technological system, trad. du français par J. Neugroschel, New York, Continuum.

Faulkner W., 2000, «The power and the pleasure? A research agenda for making gender stick to engineers », Science, Technology \& Human Values, vol. 25, I, pp. 87- I 9.

Giddens A., 1984, The constitution of society, Cambridge, Polity.

ITU (International Telecommunication Union). ICT Statistics. Accès : http ://www.itu.int/ ITU-D/ict/statistics/ict/graphs/internet.jpg. Consulté le 07/07/09.

Katz J., Aspden P., 1998 « Internet dropouts in the USA », Telecommunications Policy, 22, 4/5, pp. 327-339.

Kline R., 2003, « Resisting consumer technology in rural America: The telephone and electrification », pp. 51-66, in: Oudshoorn N., Pinch T., eds, How users matter: The co-construction of users and technology, Cambridge, MA, MIT Press.

Latour B., 2005, Reassembling the social. An introduction to actor-network-theory, Oxford, Oxford University Press.

Rainie L., Madden M., Boyce, A., Lenhart A., Horrigan J., Allen K., O'Grady E., 2003, «The ever-shifting Internet population: A new look at Internet access and the digital divide, Pew Internet and American Life Project 》. Accès: http ://www.pewinternet.org/ PPF/r/88/report_display.asp. Consulté le 03/03/2008.

Lie M., 2003, He, she and IT revisited. New perspectives on gender and the information society, Oslo, Gyldendal.

Lie M., Sørensen, K. eds, 1996, Making technology our own? Domesticating technology into everyday life, Oslo, Scandinavian University Press.

Lindsay C., 2003, « From the shadows: Users as designers, producers, marketers, distributors and technical support », pp. 29-50, in : Oudshoorn N., Pinch T., eds, How users matter: The co-construction of users and technology, Cambridge, MA, MIT Press.

Miles I., Thomas G., 1995, « User resistance to new interactive media: Participants, processes and paradigms », pp. 255-275, in : Bauer M., ed., Resistance to new technology, Cambridge, Cambridge University Press.

Nationmaster.com (compiled from OECD Communications Outlook, 2003, Tables 5.6 \& 3. 12). Web sites by country \& Communication technology patents by country. Definition, graph and map. Accès : http ://www.nationmaster.com. Consulté le 07/08/09.

Neice D., 2002, « Cyberspace and social distinctions: Two metaphors and a theory », pp. 55-84, in: Mansell R., ed., Inside the communication revolution, Oxford, Oxford University Press. 
Oudshoorn N., 2008, « Diagnosis at a distance: The invisible work of patients and healthcare professionals in cardiac telemonitoring technology », Sociology of Health \& Illness, vol. 30, 2, pp. 272-288.

Oudshoorn N., Pinch T., eds, 2003, How users matter: The co-construction of users and technology, Cambridge, MA, MIT Press.

Riehm U., Krings B.-J., 2006, « Abschied vom "Internet für alle"? Der "blinde Fleck" in der Diskussion zur digitalen Spaltung », Medien \& Kommunikationswissenschaft, 54, I, pp. 75-94.

Rogers E., 1986, Communication technology. The new media in society, New York, Free Press.

Selwyn N., Gorard S., Furlong J., 2005, « Whose internet is it anyway? Exploring adults (non)use of the internet in everyday life », European Journal of Communication, vol. 20, I, pp. 5-26.

Silverstone R., Hirsch E., eds, 1992, Consuming technologies: Media and information in domestic spaces, London, Routledge.

Thomas G., Wyatt S., 2000, « Access is not the only problem : using and controlling the Internet », pp. 2I-45, in :Wyatt S., Henwood F., Miller N., Senker P., eds, Technology and in/equality: Questioning the information society, London, Routledge.

Turkle S., 1995, Life on the screen. Identity in the age of the internet, New York, Simon \& Schuster.

Uotinen J., 2003, « Involvement in (Information) Society - The Joensuu Community Resource Centre Netcafé », New Media \& Society, vol. 5, 3, pp. 335-56.

Wajcman J., 2004, Technofeminism, Cambridge, Polity Press.

Woolgar S., 2002, « Five rules of virtuality », pp. 1-22, in : Woolgar S., ed. Virtual society? Technology, cyberbole, reality, Oxford, Oxford University Press.

Wyatt S., 1999, «They came, they surfed, they went back to the beach: why some people stop using the internet », prepared for the Society of Social Studies of Science conference, San Diego, October. Accès : http ://virtualsociety.sbs.oc.ac.uk/reports/surf. htm. Consulté le 10/07/08.

- 2003, « Non-users also matter: The construction of users and non-users of the Internet », pp. 67-79, in : Oudshoorn N.,PinchT., eds, How users matter :The co-construction of users and technology, Cambridge, MA, MIT Press.

Wyatt S., Henwood F., Hart A., Smith J., 2005, «The digital divide, health information and everyday life », New Media and Society, vol. 7, 2, pp. 199-218.

Wyatt S., Thomas G.,Terranova T., 2002, «They came, they surfed, they went back to the beach : Conceptualising use and non-use of the Internet », pp. 23-40, in :Woolgar S., ed. Virtual society? Technology, cyberbole, reality, Oxford, Oxford University Press. 